\title{
A Study of Purchase Intention of Eco-friendly Products: A Cross-Cultural Investigation between Korea and China
}

\author{
Jiyoung Yoon ${ }^{1}$ and Soonhee Joung ${ }^{2}$ \\ ${ }^{1}$ Adjunct Professor, Department of Consumer Studies, \\ Ewha Woman's University, Korea \\ ${ }^{2}$ Professor, Department of Consumer Studies, Ewha Woman's University, Korea \\ Igraceyoon928@ewha.ac.kr, ${ }^{2} j$ sh@ewha.ac.kr
}

\begin{abstract}
This study sought to compare the purchase intention of eco-friendly products between Korean and Chinese consumers. For this purpose, it established a conceptual model in which environmental knowledge affects attitude, subjective norm, and perceived behavioral control toward eco-friendly products, which in turn are connected to the purchase intention of ecofriendly products. According to the analysis results, in both Korean and Chinese cases, consumers' environmental knowledge had a positive effect on their attitude to eco-friendly products and subjective norms, and the variable that exerted the strongest influence on purchase intention was the subjective norm. On the other hand, this research found some differences in the results between the two countries: in the Korean case, the path from perceived behavioral control to purchase intention was not statistically supported, whereas in the Chinses case the path from environmental knowledge to perceived behavioral control was rejected. ${ }^{I}$
\end{abstract}

Keywords: Theory of planned behavior, An extended theory of planned behavior, Ecofriendly products, Environmental knowledge, Purchase intention, Korea, China, Comparative culture

\section{Introduction}

In the summer of 2018, South Korea recorded the largest number of 'heat wave' days since 1973, in which the statistics of meteorological observations started being made [1]. As environmental abnormalities like this have emerged all over the world, the crisis awareness of the global environment has been rising. In addition, the need for environmental behavior for the survival of the present and future generations has been emphasized.

Korean consumers are also more concerned about environmental consumption than in the past. However, looking into the statistical numbers, we can see that the percentage that environmental concerns lead to purchase behavior of eco-friendly products is still low [2][3]. On the other hand, in some studies carried out in Europe, in which environmental education has been actively conducted, more than $70 \%$ of the study participants had high levels of crisis awareness of environmental problems, and nearly two-thirds of them were purchasing ecofriendly products [4][5]. The reason that such difference in environmental behavior occurs among countries may be due to the differences in the level of consciousness of individuals, but it may also be that the degree of understanding of eco-friendly consumption varies among

Article history:

Received (September 21, 2019), Review Result (October 17, 2019), Accepted (November 12, 2019) 
countries. That is, consumers' understanding of eco-friendly products may vary among countries, because of differences in the economic and education levels and culture. Environmental problems cannot simply be identified as problems of individual countries, so cross-national studies need to be conducted on the problems. However, most studies on ecofriendly issues surveyed consumers of one country rather than identifying differences between countries [6][3]. Therefore, we conducted a study of purchase intention with Korean and Chinese consumers. It would be meaningful to compare Chinese consumers to Korean consumers, reflecting the recent changes in China, which has shown great interest in environmental issues, such as enacting the environmental law in 2015.

To this end, this study established a theoretical model by applying the theory of planned behavior, which enables comprehensive study on antecedent factors human behavior, and suggested a model of 'an extended theory of planned behavior'. The antecedent variable [7] was environmental knowledge, which has been known to lead to the change of environmental attitude through prior studies.

The purpose of this study was to conduct empirical tests on the proposed framework of the process toward the intent of purchasing eco-friendly products and to examine differences in processes between Korean and Chinese consumers.

\section{Literature review}

\subsection{Theory of planned behavior}

To explain human behavior, Ajzen suggested attitude, subjective norm, and perceived behavioral control as the antecedent variables, and established the theory of planned behavior which postulates that those three variables affect human behavior intention [7]. Furthermore, this scholar stated that it would be possible to add necessary variables or modify the paths to enhance the power of explanation of the theory [7]. Thus, we aimed to examine the process of consumers' purchase intention of eco-friendly products by including environmental knowledge. And it turned out to exert a significant effect on the existing variables of the theory of planned behavior.

\subsection{Environmental knowledge}

In this study, environmental knowledge is information for understanding and evaluating human behavior that can influence the global environment, and refers to general knowledge in the ecosystem [8]. Individuals with a high environmental knowledge are highly interested in the seriousness of environmental problems, take environmental actions, and make positive changes in environmental attitude as well as eco-friendly actions [9]. Therefore, this study included environmental knowledge.

\section{Conceptual model for this research}

This study suggested six hypotheses by applying the theory of planned behavior. Moreover, it examined differences in the same paths between Korean and Chinese consumers.

H1. Environmental knowledge will have a positive effect on attitude toward eco-friendly clothing.

H2. Environmental knowledge will have a positive effect on subjective norms toward ecofriendly clothing. 
H3. Environmental knowledge will have a positive effect on perceived behavioral control toward eco-friendly clothing.

H4. Attitude toward eco-friendly clothing will have a positive effect on the purchase intention of the products.

H5. Subjective norms toward eco-friendly clothing products will have a positive effect on the purchase intention of the products.

H6. Perceived behavioral control toward eco-friendly clothing products will have a positive effect on the purchase intention of the products.

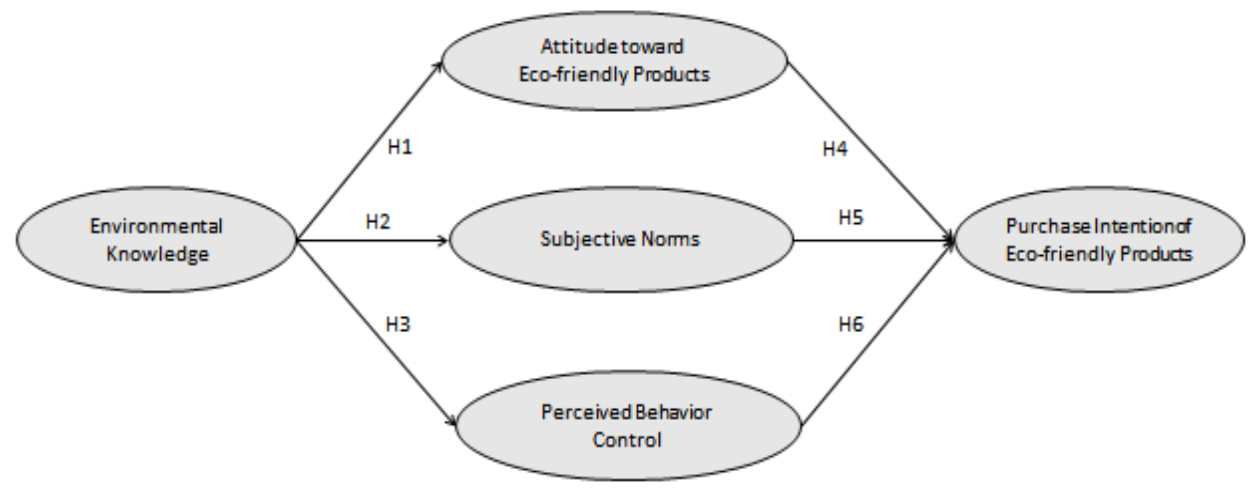

Figure 1. The conceptual model

Table 1. The survey items for all the variables

\begin{tabular}{|c|c|}
\hline Constructs & Items \\
\hline \multirow{4}{*}{$\begin{array}{c}\text { Environmental } \\
\text { knowledge }\end{array}$} & I am very knowledgeable about environmental issues. \\
\cline { 2 - 3 } & I know how to select products and packages that reduce the number of waste ending \\
landfills.
\end{tabular}




\section{Methodology}

For this study, data was collected from female college students in their 20s. College students of the age group have comparatively similar characteristics in terms of socio-economic status among countries [10]. The eco-friendly products selected for this study were clothing - the shopping category in which women in their twenties take the greatest interest [11].

For this research, in South Korea, an online survey was conducted with female college students residing in Seoul, and in China, the survey was administered through an online survey site - that is, Wenjuanxing - with female college students residing in Beijing. This study used the same survey questions for the two cases. The final questionnaire was further back-translated in Korean and English and reviewed by bilingual scholars. All the survey questions are based on prior studies by a 7-point Likert scale. An exclusion criterion, in the final analysis, included non-response and age and sex not covered by the limitations of the online survey.

We collected 236 and 315 completed questionnaires in South Korea and China, respectively, and used them for the statistical analyses. For each variable, we modified the survey items validated and used by a prior study to make them suitable for the Korean and Chinese contexts. The final analyses included 5 survey items for environmental knowledge [5], 5 for attitude [12], 3 for subjective norm [12], 4 for perceived behavioral control [13], and 3 for purchase intention [13] [Table 1]. According to the reliability test results, all the measures showed a satisfactory level of reliability, having a reliability coefficient (Cronbach's $\alpha$ ) greater than .80 .

\section{Data analysis result}

This study tested the suggested hypotheses by using the SPSS and AMOS statistical programs.

According to the results of the SEM (Structural Equation Modelling), both countries showed satisfactory levels of model fit indices. In the Korean case, except for one hypothesis (regarding PBC $\rightarrow$ PI path), the other five were supported. In the Chinese case, only one hypothesis (regarding the $\mathrm{EK} \rightarrow \mathrm{PBC}$ path) of the six was not statistically supported. In a nutshell, in both cases, only one hypothesis was rejected but the rejected hypothesis was different between the two cases. That is, we found differences between Korea and China Table 2.

Table 2. The results of the structural equation modeling

\begin{tabular}{|c|c|c|c|c|c|c|c|c|c|}
\hline \multirow[b]{2}{*}{$\mathrm{H}$} & \multirow{2}{*}{\multicolumn{3}{|c|}{ Paths }} & \multicolumn{3}{|c|}{ South Korea } & \multicolumn{3}{|c|}{ China } \\
\hline & & & & Coefficient & $R^{2}$ & Results & Coefficient & $\mathrm{R}^{2}$ & Results \\
\hline H1 & \multirow{3}{*}{ EK } & $\rightarrow$ & AT & $0.39(3.91)^{* *}$ & 0.12 & Supported & $0.25(3.39)$ & 0.06 & Supported \\
\hline $\mathrm{H} 2$ & & $\rightarrow$ & SN & $0.26(3.58)^{* *}$ & 0.07 & Supported & $0.43(4.45)^{* *}$ & 0.19 & Supported \\
\hline $\mathrm{H} 3$ & & $\rightarrow$ & PBC & $0.53(8.12) * *$ & 0.28 & Supported & $0.17(1.90) * *$ & 0.04 & Rejected \\
\hline $\mathrm{H} 4$ & AT & $\rightarrow$ & \multirow{3}{*}{ PI } & $0.19(4.11)^{* *}$ & 0.04 & Supported & $0.28(3.42) * *$ & 0.08 & Supported \\
\hline H5 & SN & $\rightarrow$ & & $0.57(8.78) * *$ & 0.30 & Supported & $0.49(4.81)^{* *}$ & 0.24 & Supported \\
\hline H6 & PBC & $\rightarrow$ & & $-0.05(-1.24)$ & 0.00 & Rejected & $0.31(3.30)^{* *}$ & 0.10 & Supported \\
\hline
\end{tabular}

\section{Discussion and conclusion}

This comparative study is on the purchase intention of eco-friendly clothing products between two countries. To this end, the present research suggested a model of an extended theory of planned behavior that had environmental knowledge as the antecedent variable and examined differences in the full process between Korean and Chinese. According to test results 
of the suggested hypotheses, in both the Korean and Chinese cases environmental knowledge turned out to have a positive effect on subjective norm and attitude. As environmental knowledge increased, the eco-friendly attitude was likely to more positive change and ecofriendly subjective norms become stronger. Thus, it can be expected that environmental knowledge education is highly likely to lead to eco-friendly purchase intention in both countries.

On the other hand, we found a few differences between the Korean and Chinese cases. In the Korean case, of the six hypotheses, only the hypothesis expected a positive association. In the Chinese case, solely the hypothesis concerning the positive relationship between environmental knowledge and perceived behavioral control was rejected. The findings of this research show that through environmental knowledge education both Korea and China are highly likely to lead to changes in eco-friendly purchasing processes. In particular, it is expected that if subjective norm elicits changes that socially call for eco-friendly behavior, more consumption will lead to a higher percentage of eco-friendly purchasing.

\section{References}

[1] Korea Metrological Administration, (2018), http://www.kma.go.kr

[2] Statistics Kore, a (2016), http://kostat.go.kr

[3] Y. Jiyoung, "A study on an eco-friendly product purchase process using the expanded theory of planned behavior," Ewha Womans University, (2019)

[4] C. B. Hanson, "Environmental concern, attitude toward green corporate practices, and green consumer behavior in the United States and Canada," American Society of Business and Behavioral Sciences eJournal, vol. 9, no. 1, pp.62-70, (2013)

[5] K. Seung Bong and J. Byoungho, "Predictors of purchase intention toward green apparel products: A crosscultural investigation in the USA and China," Journal of Fashion Marketing and Management: An International Journal, vol.21, no.1, pp.70-87, (2017)

[6] Y. S. Chen, S. B. Lai, and C.T. Wen, "The influence of green innovation performance on corporate advantage in Taiwan," Journal of Business Ethics, vol.67, no.4, pp.331-339, (2006)

[7] I. Ajzen, "The theory of planned behavior organizational behavior and human decision Processes," vol.50, pp.179-211

[8] G. E. Fryxell and C. W. H. Lo, "The influence of environmental knowledge and values on managerial behaviors on behalf of the environment: An empirical examination of managers in China," Journal of Business Ethics, vol.46, no.1, pp.45-69, (2003)

[9] F. G. Kaiser, S. Wolfing, and U. Fuhrer, "Environmental attitude and ecological behavior," Journal of Environmental Psychology, vol.19, no.1, pp.1-19

[10] R. A. Peterson, "On the use of college students in social science research: Insights from a second-order metaanalysis," Journal of Consumer Research, vol.28, no.3, pp.450-461, (2001)

[11] R. Gardyn, "Educated consumers,” American Demographics, vol.24, no.10, pp.18-19, (2002)

[12] C. J. Armitage and M. Conner, "Efficacy of the theory of planned behavior: A meta-analytic review," British Journal of Social Psychology, vol.40, pp.471-499, (2001)

[13] J. Paul, A. Modi, and J. Patel, "Predicting green product consumption using the theory of planned behavior and reasoned action," Journal of Retailing and Consumer Services, March 29, pp.123-134, (2016) 
A Study of Purchase Intention of Eco-friendly Products: A Cross-Cultural Investigation between Korea and China

This page is empty by intention. 